\title{
Excitation Spectrum of One-dimensional Extended Ionic Hubbard Model
}

\author{
M. Hafez ${ }^{* 1}$ and S. A. Jafari ${ }^{\dagger 2,3,4}$ \\ ${ }^{I}$ Department of Physics, Tarbiat Modares University, Tehran, Iran \\ ${ }^{2}$ Department of Physics, Isfahan University of Technology, Isfahan 84156-83111, Iran \\ ${ }^{3}$ Department of Physics, Sharif University of Technology, Tehran 11155-9161, Iran \\ ${ }^{4}$ School of Physics, Institute for Research in Fundamental Sciences (IPM), Tehran 19395-5531, Iran
}

\begin{abstract}
We use Perturbative Continuous Unitary Transformations (PCUT) to study the one dimensional Extended Ionic Hubbard Model (EIHM) at half-filling in the band insulator region. The extended ionic Hubbard model, in addition to the usual ionic Hubbard model, includes an inter-site nearest-neighbor (n.n.) repulsion, $V$. We consider the ionic potential as unperturbed part of the Hamiltonian, while the hopping and interaction (quartic) terms are treated as perturbation. We calculate total energy and ionicity in the ground state. Above the ground state, (i) we calculate the single particle excitation spectrum by adding an electron or a hole to the system. (ii) the coherence-length and spectrum of electron-hole excitation are obtained. Our calculations reveal that for $V=0$, there are two triplet bound state modes and three singlet modes, two anti-bound states and one bound state, while for finite values of $V$ there are four excitonic bound states corresponding to two singlet and two triplet modes. The major role of on-site Coulomb repulsion $U$ is to split singlet and triplet collective excitation branches, while $V$ tends to pull the singlet branches below the continuum to make them bound states.
\end{abstract}

PACS numbers: 71.35.-y, 71.30.+h, 71.10.Li

\section{INTRODUCTION}

The ionic Hubbard model was introduced to describe the Neutral-Ionic transition in the charge-transfer organic mixedstack compounds such as TTF-Chloranil ${ }^{1}$. The transition occurs by change in pressure or temperature of these materials $s^{2.3}$. Since then, in addition to experimental studies ${ }^{4.5}$, some theoretical investigations for identifying the nature of possible phases and optical properties have been conducted on this model $\underline{6}^{6}$ and its extended versions $\frac{7.8}{}$.

On the other hand, in transition metal oxides $\mathrm{BaTiO}_{3}$, $\mathrm{KNbO}_{3}$, and $\mathrm{KTaO}_{3}$ it was noted that the valence of the transition metal ion is $\sim+3^{9.10}$, rather than +4 which is expected if a simple ionic picture holds. Therefore it is necessary to consider effects of strong correlations in these materials, leading to the so called ionic Hubbard model (IHM) $\stackrel{11}{ }$. Although the phase transitions at zero $\frac{12-17}{17}$ and finite $\frac{18-20}{2}$ temperatures for this model have been studied by many researchers, the physical properties of band-insulating regime of this model has not been studied much $^{21}$.

Besides Hubbard-Peierls Hamiltonian ${ }^{22}$, the ionic Hubbard model is also a candidate for Halogen-bridged transitionmetal compounds ${ }^{21}$ known as MX-chains, where $\mathrm{M}$ stands for transition-metal and $\mathrm{X}$ for Halogen. There are many theoretical and experimental researches on the optical properties of these compounds. In NiX-chains, due to large on site interaction, the system is Mott insulator while PdX- and PtX-chains are band insulators $23-25$.

\footnotetext{
*Electronic address: m.hafeztorbati@yahoo.com

${ }^{\dagger}$ Electronic address: jafari@sharif.ir
}

The one dimensional EIHM Hamiltonian is as follows:

$$
\begin{aligned}
H= & \frac{\Delta}{2} \sum_{i, \sigma}(-1)^{i} n_{i, \sigma}+\frac{t}{4} \sum_{i, \sigma}\left(c_{i, \sigma}^{\dagger} c_{i+1, \sigma}+\text { h.c. }\right) \\
& +U \sum_{i} n_{i, \uparrow} n_{i, \downarrow}+\frac{V}{2} \sum_{i} n_{i} n_{i+1},
\end{aligned}
$$

where $\Delta$ is one-particle ionic potential, $t / 4$ is nearestneighbor hopping amplitude, $U$ represents on site repulsive interaction, and $V / 2$ is nearest-neighbor inter-site interaction. $c_{i, \sigma}^{\dagger}$ and $c_{i, \sigma}$ are electron creation and annihilation operators at site $i$ with spin $\sigma$, respectively. $n_{i, \sigma} \equiv c_{i, \sigma}^{\dagger} c_{i, \sigma}$ is the electron occupation operator and $n_{i} \equiv \sum_{\sigma} n_{i, \sigma}$. When $\Delta$ is dominant energy scale, i.e. $\Delta \gg t, U, V$ the ground state of this model is a band insulator, with electrons and holes and their possible (anti)bound-states constituting the excitation spectrum. When $U$ dominates, i.e. $U \gg t, \Delta, V$ the ground state is Mott insulator, with doublon and holon excitation. In this paper, we investigate the one dimensional EIHM by PCUT method in the band insulator region. We take the first term in Eq. (1) as unperturbed Hamiltonian, and the rest as perturbation. In the following, PCUT method is introduced. Then by using this method, ground state energy, ionicity, spin and charge gaps, one electron and one hole dispersion, as well as spectrum and coherence length of the electron-hole excitation for EIHM are calculated.

\section{PCUT METHOD}

PCUT or perturbative flow equation method is a high order perturbative approach for calculating the multi-particle excitations and observables in model Hamiltonians ${ }^{26-29}$. In this method, first the Hamiltonian is mapped, by flow equations approach ${ }^{30}$, to a perturbative effective Hamiltonian. This effective Hamiltonian is obtained using a generator ${ }^{31}$ which 
conserves the number of quasi-particles that are the excitations of unperturbed part. Then by using linked cluster expansion theorem ${ }^{32}$, physical quantities in the thermodynamic limit can be calculated employing finite size clusters. To be self-contained, here we concisely review PCUT method.

Assume that the Hamiltonian can be written as a perturbative problem:

$$
H=Q+x T
$$

where $x T$ is the part of the Hamiltonian, the effect of which we would like to consider perturbatively. In order to apply PCUT method on the Hamiltonian (2), $Q$ is required to have equi-distance spectrum. The difference between two successive levels is chosen as the unit of energy. Also $T$ must be of the following form:

$$
T=\sum_{n=-N}^{+N} T_{n}
$$

where $N$ is a finite number, and $T_{n}$ is a ladder operator which decreases or increases the eigen-values of $Q$ by $n$ units.

When these conditions are satisfied, the flow equation and the quasi-particle conserving generator respectively read,

$$
\begin{gathered}
\partial_{\ell} H(\ell)=[\eta(\ell), H(\ell)], \\
\eta_{i, j}(\ell)=\operatorname{sign}\left(Q_{i}-Q_{j}\right) H_{i, j}(\ell),
\end{gathered}
$$

where $Q_{i}$ is the $i$ th eigen value of $Q$. These equations can be used to transform the Hamiltonian (2) to the following effective Hamiltonian,

$$
H_{\mathrm{eff}}=Q+\sum_{k=1}^{\infty} x^{k} \sum_{|\vec{m}|=k, M(\vec{m})=0} C(\vec{m}) T(\vec{m})
$$

where $|\vec{m}|=k$ means that $\vec{m}$ is a vector of order $k, T(\vec{m})=$ $T_{m_{1}} \cdots T_{m_{k}}, M(\vec{m})=m_{1}+\cdots+m_{k}$, and the summation is over the components of $\vec{m}$ which can take values $-N,-N+1 \cdots+N$. The $C(\vec{m})$ are known as fractional coefficients which can be obtained from a set of coupled nonlinear first order differential equations. Uhrig and his group, who formulated PCUT method, calculated these coefficients up to high orders which are available, electronically 33 . This part of the method is quite generic and holds for all Hamiltonians that fulfill the above conditions. The salient feature of the PCUT effective Hamiltonian is the constraint $M(\vec{m})=0$. This constraint causes the effective Hamiltonian to connect only the states of $Q$ corresponding to same eigen-values. Therefore, interpreting the excitations of $Q$ as quasi-particles (QPs), it is sufficient to diagonalize the effective Hamiltonian only in sectors with definite number of quasi-particles, e.g. in 0-, 1-, and 2-particle sectors. This provides a picture of the low lying excitation spectrum of the system.

In order to calculate the matrix elements of $H_{\text {eff }}$ for an infinite lattice, first it is necessary to decompose the effective Hamiltonian to irreducible $n$-particle operators that are cluster additive ${ }^{27}$. Only for these operators, the matrix elements calculated for finite size are valid in thermodynamic limit, i.e. there would be no finite size error. Therefore in the next step one needs to identify minimum cluster size along with suitable form of boundary conditions to calculate required matrix elements ${ }^{28}$. Note that despite the generic part of the method, the choice of cluster depends on the properties of the specific Hamiltonian at hand.

PCUT method has been extensively used in spin systems in order to calculate ground state energy, 1-particle dispersion, 2particle excitation spectrum, and observables $34-40$. However, to our knowledge, this method has not been applied much to fermionic systems ${ }^{41}$. In this paper the aim is to implement this method on EIHM Hamiltonian in order to calculate ground state energy, 1-electron and 1-hole dispersions, as well as spectrum and coherence-length of electron-hole excitation. We use Padé approximation with polynomials of various orders to enlarge the convergence radius of the obtained perturbative expansions.

\section{APPLICATION OF PCUT METHOD TO EIHM}

To implement PCUT method on EIHM, we write this Hamiltonian as Eq. (2). Now we identify $x=t$ and,

$$
\begin{aligned}
Q= & \frac{\Delta}{2} \sum_{i, \sigma}(-1)^{i} n_{i, \sigma}, \\
T= & \frac{1}{4} \sum_{i, \sigma}\left(c_{i, \sigma}^{\dagger} c_{i+1, \sigma}+\text { h.c. }\right)+u \sum_{i} n_{i, \uparrow} n_{i, \downarrow} \\
& +\frac{v}{2} \sum_{i} n_{i} n_{i+1},
\end{aligned}
$$

where $u \equiv \frac{U}{t}$, and $v \equiv \frac{V}{t}$. It is convenient to choose $\Delta$ as energy unit and set $\Delta=1$. Note that the popular convention for the unit of energy in Hubbard model literature is to take $t$ as unit of energy. Moreover, a different sign convention for $t$ is used in the literature. This should be noted when comparing our results with other references. It is obvious that at half-filling, $Q$ has an equi-distance spectrum and its energy difference between two successive levels is 1 . Therefore the first condition is satisfied. The exact ground state of $Q$ corresponds to a configuration where all odd sites are doubly occupied and all even sites are left empty. Since there are no electrons in even sites, nor holes in odd sites, in the sense of having no quasi-particles, one can interpret the ground state as vacuum. The excitations of $Q$ can be obtained by moving an electron from odd site to an even site. Or alternatively in terms of quasi-particle picture, when an electron is added to even sites and simultaneously a hole is added to odd sites. This is a 2-particle excitation that includes two types of particles. For obtaining the properties of $H_{\text {eff }}$ in this sector we must first discuss 1-particle sectors corresponding to a single electron (hole) added to even (odd) sites. These one particle sectors themselves include very important physical information like electron and hole dispersions, which can be directly measured in (inverse) photo-emission spectroscopies. 
Since the eigen-states of $Q$ can be used as basis for $H$, it is possible to write $T$ in the form of Eq. (3). The interaction terms in Eq. (8) compose $T_{0}$ and with some simple calculations we can decompose hopping term to $T_{-1}$, and $T_{+1}$. Therefore second condition is satisfied with $N=1$, which reflects the fact that hopping is associated with a pair of particle-holes. These operators are defined as $T_{n}=\sum_{i} \Gamma_{n}(i)$, where summation is over lattice points and $\Gamma_{n}(i)$ only connects nearest-neighbor sites, i.e. $i$ and $i+1$. The $\Gamma_{n}(i)$ are defined as:

$$
\begin{aligned}
& \Gamma_{0}(i)=u n_{i, \uparrow} n_{i, \downarrow}+\frac{v}{2} n_{i} n_{i+1}, \\
4 \Gamma_{+1}(i)= & |0, \uparrow\rangle\langle\uparrow, 0|-| \uparrow, d\rangle\langle d, \uparrow|-| \downarrow, d\rangle\langle d, \downarrow| \\
+ & |0, d\rangle\langle\uparrow, \downarrow|-| \downarrow, \uparrow\rangle\langle d, 0|+| \uparrow, \downarrow\rangle\langle d, 0| \\
+ & |0, \downarrow\rangle\langle\downarrow, 0|-| 0, d\rangle\langle\downarrow, \uparrow| \quad, \quad i \in \text { odd,(10) }
\end{aligned}
$$

where $|a, b\rangle$ in the above notation means $|a\rangle_{i} \otimes|b\rangle_{i+1}$, and $|0\rangle$, $|\downarrow\rangle,|\uparrow\rangle$, and $|d\rangle$ stand for a site occupied with no, spin down, spin up, and two electron(s), respectively. The above term is essentially a right to left hopping, which has been expanded in terms of Hubbard operators for practical purposes. We also have $\Gamma_{+1}(i \in$ even $)=\Gamma_{+1}^{\dagger}(i \in$ odd $), \Gamma_{-1}(i \in$ even $)=$ $\Gamma_{+1}(i \in$ odd $)$, and $\Gamma_{-1}(i \in$ odd $)=\Gamma_{+1}(i \in$ even $)$. In writing Eq. (10) we have used the following sign convention for Fermi operators: When $c_{i, \sigma}$ or $c_{i, \sigma}^{\dagger}$ act on a state a phase factor $(-1)^{S_{i, \sigma}}$ is produced, where $S_{i, \sigma}$ denotes the total (both $\uparrow$ and $\downarrow$ ) number of electrons located in the left side of site $i$. Moreover, at any given site $i$, spin- $\uparrow$ electron always appears to the left side of spin- $\downarrow$ electron. As long as one is interested in one dimensional lattices the choice of suitable clusters to find the irreducible interactions is simple while it becomes a tricky task in higher dimensions and for lattices with complicated structures. It is obvious from Eqs. (9) and (10) that when $\Gamma_{ \pm 1}(i)$ acts on two nearest-neighbor sites, it always transfers the quasi-particles. But $\Gamma_{0}(i)$ acts on two nearestneighbor sites with no transfer of QPs. When we calculate 1-particle hopping terms and 2-particle interactions in thermodynamic limit based on full graph theory $\underline{28}$, this property of $\Gamma_{0}(i)$ in EIHM, in analogy with models involving 4-spin interactions $s^{42}$, generates larger clusters compared to systems with solely 2-spin interactions perturbed around the dimer limit ${ }^{26,28}$.

\section{A. Ground state energy and ionicity}

Since $H_{\text {eff }}$ and $Q$ have the same ground state (unless a

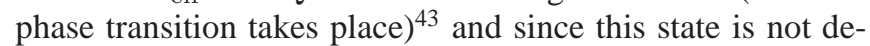
generate, the ground state energy of $H$ per site is given by $\epsilon_{0}=\frac{1}{L}\left\langle 0\left|H_{\text {eff }}\right| 0\right\rangle$, where $|0\rangle$ is the ground state of $Q$ or the complex vacuum. We have calculated $\epsilon_{0}$ up to order 9 in $t, U$, and $V$. At this order, from Eqs. (9) and (10) it turns out that the minimum cluster size is 10 with periodic boundary condition to obtain the thermodynamic limit value of $\epsilon_{0}$. Ground state energy per site is given in appendix. We have plotted ground

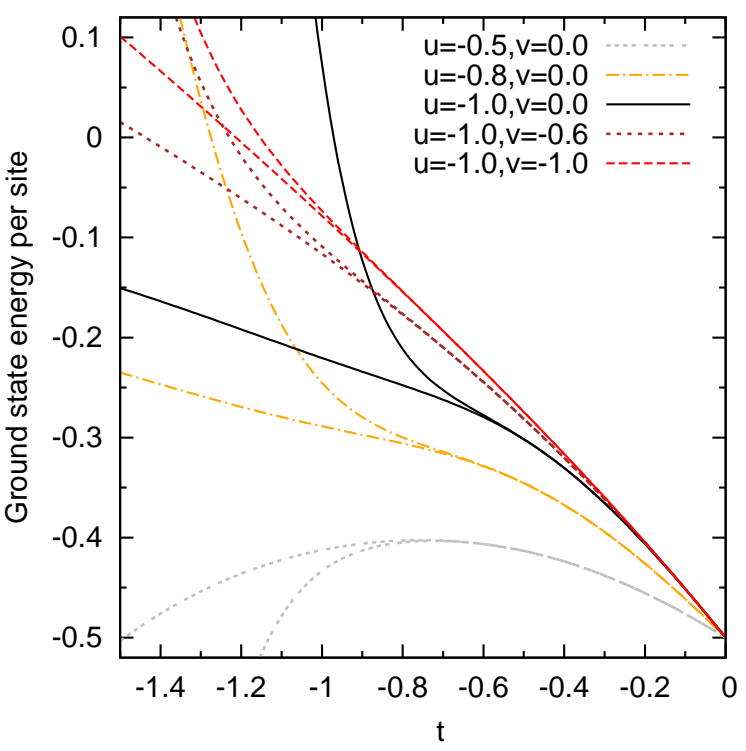

FIG. 1: Ground state energy per site versus $t$ for $\Delta=1$ and different values of $u$ and $v$. In each parameter set both plain series and the best Padé approximation have been depicted. The plain series expansions diverge at some special values of $t$ while the Padé approximant are converged in the plotted region.

state energy per site versus $t$ for different values of dimensionless interaction parameters $u$ and $v$ in Fig. 11 Note that in this figure, each graph represents the best Padé approximation. It is seen form Fig. 1 that ground state energy increase by increasing $U$ and $V$. This is physically plausible, as repulsive interactions always increase energy of the system, unless a phase transition occurs. Note that since in our convention, electron-like bands are represented with negative values of $t$, the negative values of $u, v$ in Fig. 1 correspond to repulsive interactions $U, V>0$.

From the analytical expression for ground state energy, we can use the Hellman-Feynman theorem to calculate the ionicity per site which is an important quantity for the ionic Hubbard model and is given by 14 :

$$
I \equiv-\frac{1}{L} \sum_{i, \sigma}(-1)^{i} n_{i, \sigma}=-2 \frac{\partial \epsilon_{0}}{\partial \Delta} .
$$

The ionicity per site versus $t$ for different values of $u$ and $v$ has been plotted in Fig. 2 In this figure, only the best Padé approximation have been depicted. This figure shows that as $U$ is increased the ionicity decreases while increment in $V$ increases the ionicity. Therefore the on-site Coulomb interaction $U$ tends to decrease the valence. However, the next n.n. term $V$ has the opposite effect. This behavior of $V$ is expected, as it stabilizes configurations in which n.n. sites have opposite charges with respect to the half-filled background; i.e. one is doubly occupied, with its n.n. site being empty.

If we regard Eq. (20) for $\epsilon_{0}$ as a series expansion in $t$, the coefficient of $t^{2}$ can be summed as a geometric series, such that the ground state energy per site up to order $t^{2}$ in band 


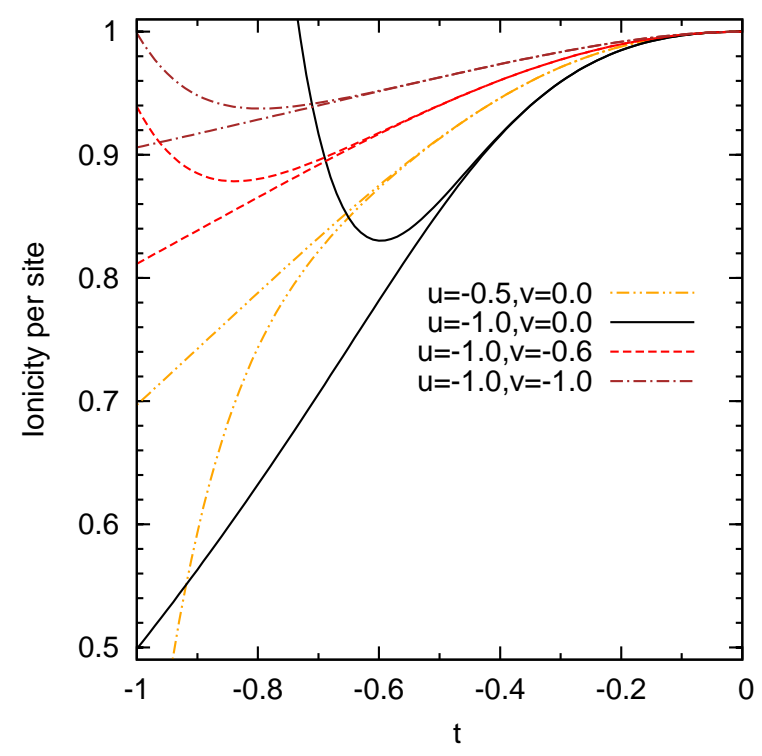

FIG. 2: Ionicity per site versus $t$ for $\Delta=1$ and different values of $u$ and $v$. In each case two graphs corresponding to the best Padé approximation and the plain series have been plotted. The plain series expansions diverge at some special values of $t$. The ionicity per site decreases (increases) by increasing in $U(V)$.

insulator phase is,

$$
\epsilon_{0}=\frac{U-\Delta}{2}-\frac{1}{8} \frac{t^{2}}{\Delta+\frac{3}{2} V-U}+O\left(t^{4}\right) .
$$

Convergence radius for this expression is obviously given by $\Delta>|3 V / 2-U|$. This expression can be interpreted as follows: In the band insulating regime, $\Delta \gg U, V$, when $t=0$ the ground state belongs to subspace of configurations in which odd sites are doubly occupied. By turning on the perturbation $t$, a second order energy gain can be obtained when an electron virtually hops to even neighboring site. From above expression and Eq. 111, we can obtain the ionicity per site at the same order as follows,

$$
I=1-\frac{1}{4} \frac{t^{2}}{\left(\Delta+\frac{3}{2} V-U\right)^{2}}+O\left(t^{4}\right) .
$$

These are perturbative expressions for ground state energy and ionicity around atomic limit that are valid for $t \ll \Delta+\frac{3}{2} V-U$ in band insulating phase. In the Mott insulating region, similar expressions can be obtained for these quantities at $V=0$ in the limit $t \ll U-\Delta^{14}$. These expressions are derived based on the mapping of the ionic Hubbard model to the Heisenberg mode ${ }^{6}$ whose exact ground state is known in $1 \mathrm{D}$.

\section{B. Electron and hole dispersions}

To calculate one-particle dispersion, one must add an electron to the even sites of the system, while the corresponding hole must be added to odd sites. Since $H_{\text {eff }}$ only connects those states of $Q$ which have same eigen-values, it can merely transfer the electron between even sites and the hole between odd sites. Therefore it generates hopping terms of the form

$$
\begin{aligned}
H_{\mathrm{eff}} & =E_{0}+t_{0, e} \sum_{i \sigma} e_{i \sigma}^{\dagger} e_{i \sigma}+\sum_{r=2,4, \ldots} t_{r} \sum_{i \sigma} e_{i, \sigma}^{\dagger} e_{i+r, \sigma}+\text { h.c. } \\
& +t_{0, h} \sum_{i \sigma} h_{i \sigma}^{\dagger} h_{i \sigma}+\sum_{r=2,4, \ldots} t_{r} \sum_{i \sigma} h_{i, \sigma}^{\dagger} h_{i+r, \sigma}+\text { h.c., (14) }
\end{aligned}
$$

where $E_{0}$ is the ground state energy and $e_{i \sigma}^{\dagger}\left(h_{i \sigma}^{\dagger}\right)$ creates an electron (hole) with spin $\sigma$ at even (odd) site $i$. The long range hopping amplitudes $t_{r}, r=2,4, \ldots$ for electron and hole are identical up to all orders in perturbation theory. However, as for the shortest range hopping amplitude, $t_{0}$ (i.e. no hopping) only the first order of perturbative expansion for the electrons and holes are different and are related by $t_{0, e}-\mu=t_{0, h}+\mu$, where $\mu=U / 2+V$ is the chemical potential at half-filling for Eq. (1). Note that if we had started from a particle-hole symmetric version of the Hamiltonian, by replacing $n_{i \sigma} \rightarrow$ $n_{i \sigma}-1 / 2$ in $U$ and $V$ terms of Eq. (1), we would have $\mu=0$ leading to $t_{0, e}=t_{0, h}$.

The hopping coefficients $t_{r}$ in the thermodynamic limit can be calculated on a finite cluster based on linked cluster theorem $^{32}$. To calculate these coefficients, first we must determine the minimum cluster size which can give us the thermodynamic limit results. The minimum cluster size, in addition to order of perturbation, depends on the particular hopping coefficient which we would like to calculate. For example, to obtain $t_{0}$ up to order 9,13 sites and up to order 10,15 sites are needed. For calculation of $t_{2}$ up to order 9,10 , and 11 one needs 13,13 , and 15 sites, respectively. For $t_{4}, t_{6}, t_{8}$, up to order 9, 11 sites are sufficient to calculate them in thermodynamic limit. These numbers can be inferred from Eqs. (9) and (10). We have calculated electron dispersion up to order 9 . At this order there are five hopping coefficient, $t_{0}, t_{2}, t_{4}, t_{6}$, and $t_{8}$ which are independent of the spin of the electron and have been reported in appendix.

Electron and hole dispersions of the effective Hamiltonian are given by,

$$
\omega_{e(h)}(k)= \pm\left(t_{0, e(h)}+2 \sum_{n=1}^{4} t_{2 n} \cos (2 n k)\right) .
$$

In Fig. 3 we have plotted the one-particle dispersion corresponding to $t=-1.0, U=1.4, V=0$, as well as $t=-1.0$, $U=1.4, V=0.3$. Note that to obtain reliable results for the value of $U=1.4$ we have used Padé approximation. The charge gap is defined as,

$$
E_{c} \equiv E_{0}(N+1)+E_{0}(N-1)-2 E_{0}(N) .
$$

For $U=0, V=0$, we have a simple band insulator with gap magnitude $\Delta$ which has been taken to be unit of energy. In Fig. 3 the charge gap in terms of the effective Hamiltonian becomes, $\omega_{e}\left(k=\frac{\pi}{2}\right)-\omega_{h}\left(k=\frac{\pi}{2}\right)$. As can be seen for $t=$ $-1, U=1.4, V=0.0$, the gap becomes very small $\approx 0.014$, which agrees very well with corresponding DMRG value ${ }^{14}$ 


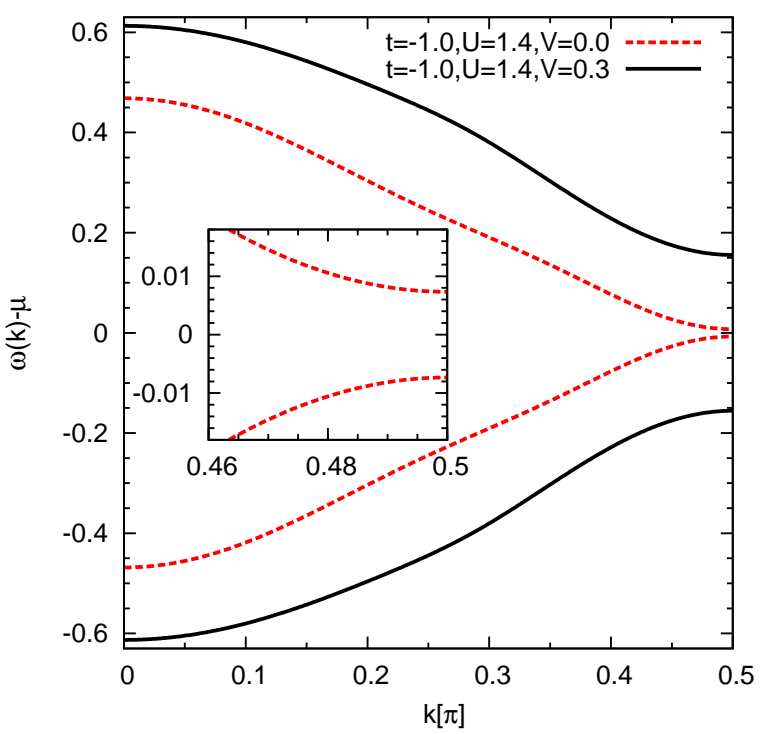

FIG. 3: Electron and hole dispersions around Fermi surface $\mu=$ $\frac{U}{2}+V$ for $(t=-1.0, U=1.4, V=0)$ and $(t=-1.0, U=1.4$, $\stackrel{V}{V}=0.3$ ) at $\Delta=1$. We have used Padé[7,2] in both cases. Our result for charge gap at $(t=-1.0, U=1.4, V=0)$ is 0.014 which is in excellent agreement with DMRG result that is 0.012 .

0.012. Note that when comparing results of Ref. 14 with ours, one has to appropriately re-scale the Hamiltonian parameters. Beyond $U \approx 1.4$ the band insulating phase terminates and one may have different behaviors for spin and charge gaps ${ }^{14}$. Although our perturbative treatment does not extend beyond the band insulating state, extension of the convergence radius captures a critical value of $U$ at which the charge gap tends to zero. Now at $U=1.4$, we turn on $V$. As can be seen in Fig. 3, turning on $V$ increases the gap.

Charge gap versus $t$ for different values of $u$ and $v$ have been plotted in Fig. 4. For each value $u, v$ the best Padé fits have been shown. For $u=-1.4, v=0$ the Padé fit shown in the figure approaches the DMRG value ${ }^{14}$ as $t \rightarrow-1$. For other values of $u, v$ reported in this figure, to ensure the quality of results, for each value $(u, v)$ we have shown two Padé fits. Comparing the variation of charge gap at $v=0$, as can be seen by moving from $u=-0.9$ (red dashed line) to $u=-1.4$ (black solid line), the gap magnitude decreases, which means Hubbard $U$ work against the band gap parameter $\Delta$ and tends to reduce it. Similarly, for a fixed value of $u$, turning on the n.n. repulsion $V=v t$ increases the band gap.

Similar to ground state energy we find expressions for $t_{0, e}$, and $t_{0, h}$, around atomic limit up to order $t^{2}$ for $V=0$. These expressions are as follows:

$$
t_{0, e}=t_{0, h}+U=\frac{\Delta}{2}+\frac{t^{2}}{8} \frac{\Delta+U}{\Delta(\Delta-U)}+O\left(t^{4}\right),
$$

and for $t_{2}$ at finite $V$ up to order $t^{2}$ we obtain:

$$
t_{2}=\frac{1}{16} \frac{t^{2}}{\Delta+V-U}+O\left(t^{4}\right) .
$$

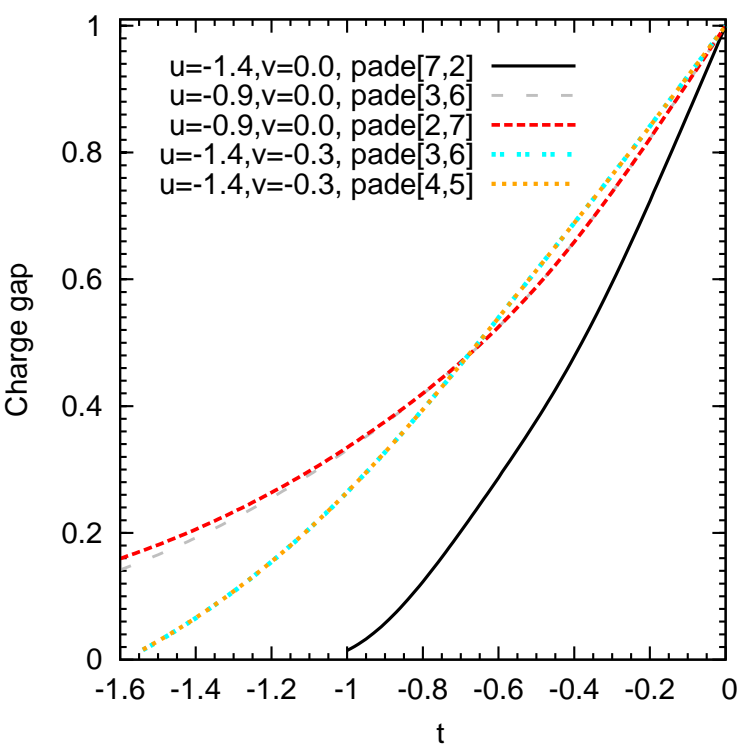

FIG. 4: Charge gap versus $t$ for different values of $u$ and $v$ in band insulator region. In all cases Padé approximation $[p, q]$ corresponding to quotient of polynomials of order $p, q$ has been used, which is indicated in the legend. Spin and charge gaps are equal, because the hopping coefficients, i.e. $t_{n}$, are independent of spin.

From these expressions we can find the electron and hole dispersions for $V=0$. The dispersions are as $\omega(k)=$ $t_{0}+2 t_{2} \cos (2 k)$. This gives rise to inverse square root density of states- a characteristic of 1D bands, the width of which is controlled by $t_{2}$.

In this subsection we obtained some informations about EIHM at half-filling by adding one electron and one hole to the system, separately. This gave rise to the band dispersion relation of the system. Now let us study the particle-hole (charge neutral) excitations of the system.

\section{Spectrum and coherence length of electron-hole excitation}

The first excited state of $Q$ at half-filling is when one electron from an odd site with energy $-\frac{1}{2}$ is transfered to an even site with energy $+\frac{1}{2}$ or in quasi-particle language when one electron is added to even sites and one hole is added to odd sites, simultaneously. This is a 2-particle excitation which include two types of particle, i.e. electron and hole. It is possible to discuss the spectrum and coherence length of this electronhole excitation by PCUT method. State of the system in this sector can be represented as $\left|i, m_{e} ; j, m_{h}\right\rangle$ where $i$ is the even site and $j$ is the odd site at which electron and hole excitations are created, respectively. Also $m_{e}$ and $m_{h}$ are magnetic quantum numbers of electron and hole. To discuss the two-particle sector, in addition to quadratic (hopping) terms, one needs to include quartic electron-hole terms of the following form in the effective Hamiltonian,

$$
t_{i_{1}, i_{2} ; j_{1}, j_{2}} h_{i_{1}}^{\dagger} e_{i_{2}}^{\dagger} e_{j_{2}} h_{j_{1}}
$$




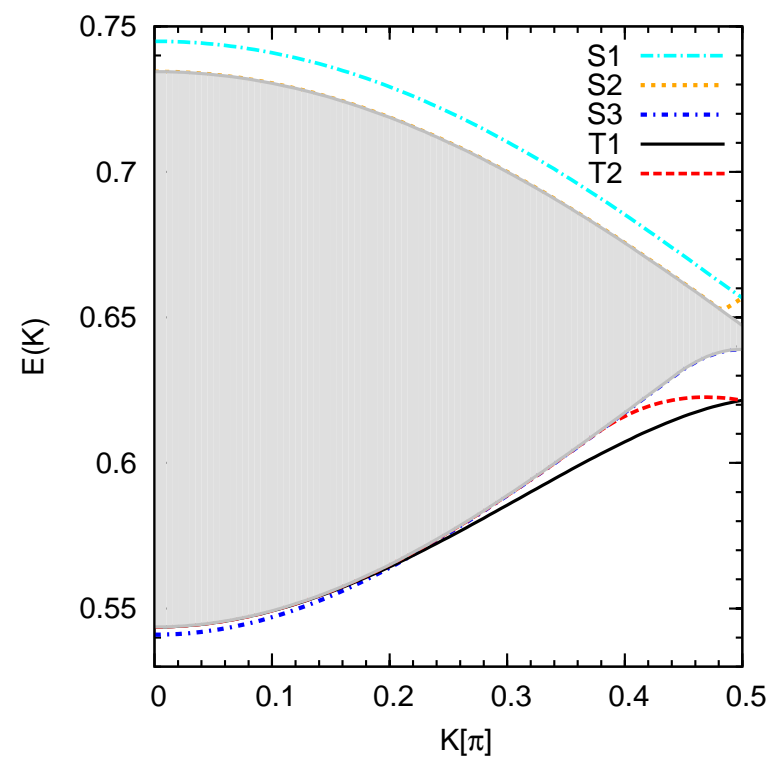

FIG. 5: Plain series result for electron-hole energy versus $K$ for $t=$ $-0.5, U=0.5$, and $V=0$. There are three singlet bound/antibound state branches and two triplet bound state branches. The two singlet anti-bound state modes and two triplet bound state modes are degenerate at $K= \pm \frac{\pi}{2}$.

Since total $H_{\text {eff }}$ preserves the total spin of the electron-hole pair, we must use the basis $|i, j ; S, M\rangle$, where $S$ is total spin of electron-hole pair and $M$ is its magnetic quantum number. $S$ can be either 0 or 1 corresponding to singlet and triplet total spin states. When $H_{\text {eff }}$ acts on states with a particle-hole pair, it gives rise to a new configuration in the subspace with a particle-hole pair. The processes of mapping the initial state to final state can be classified as 2-particle, a mixture of 1- and 2particle, or a mixture of 0-, 1-, and 2-particle process. In order to discuss this 2-particle sector, first one has to determine the irreducible 2-particle interactions obtained by subtraction of 0 - and 1- particle contributions which by linked cluster theory can be employed to calculate $t_{i_{1}, i_{2} ; j_{1}, j_{2}}$ for a thermodynamics system on a finite cluster ${ }^{27.28}$. Again similar to 1-particle sector, the minimum cluster size can be identified from properties of Eqs. (9) and (10). We have calculated these coefficients up to order 8 in $t, U, V$. These coefficients turn out to be solely dependent on total spin $S$ and not on magnetic quantum number $M$.

When the interaction terms are identified, we have only a 2particle problem that must be solved. Such a problem can be solved by changing the basis to total particle-hole momentum and their relative distance $|K, d\rangle$, or to total momentum and relative momentum $\left|K, K^{\prime}\right\rangle$ of particle-hole pair $\stackrel{44}{=}$. We use $|K, d\rangle$ representation, as in this basis it is possible to explicitly calculate electron-hole coherence length defined as ${ }^{44}$ :

$$
L=\frac{\sum_{d} f_{d}^{2}|d|}{\sum_{d} f_{d}^{2}}
$$

where $f_{d}$ is the probability amplitude for finding the particlehole pair at a relative distance $d$. In our case $d$ can take only

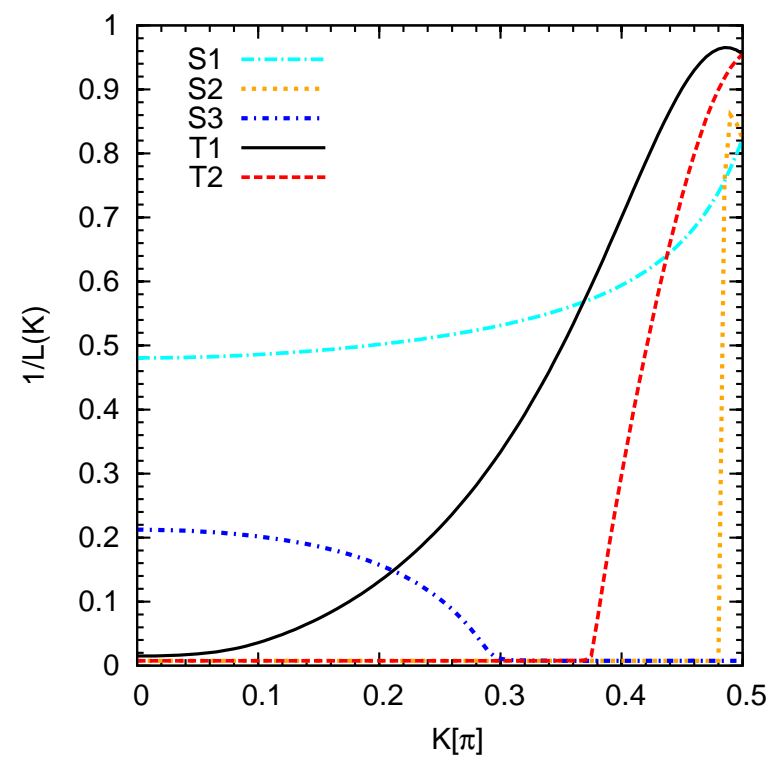

FIG. 6: Inverse of coherence length versus $K$ for $t=-0.5$, $U=0.5$, and $V=0$. For $S 1$ mode the coherence length remains finite in the whole of range of momenta. But for other modes it becomes infinite at some values of $K$. Coherence length of $S 2$ has a discontinuity at $K^{*}=0.46 \pi$.

odd values as electron are always in even sites and hole in odd sites.

Now that we have the explicit form of the $H_{\text {eff }}$ in 2-particle sector, it remains to diagonalize $H_{d, d^{\prime}}(K)$ for a given value of center of mass momentum $K$. Here $d, d^{\prime}$ indicate relative distance. For problems where the two particles are of same type (e.g. in Cooper pairing channel), this matrix is a semiinfinite ${ }^{28}$, but for problems with two types of particles, such as our case (i.e. in particle-hole channel), it is an infinite matrix. However, when dealing with these matrices on the computer, one constructs finite $N \times N$ dimensional representations of the Hamiltonian for which the eigen-value problem must be solved. Next by systematically increasing $N$, the spectrum at $N \rightarrow \infty$ limit can be obtained. For those values of $K$ where the particle-hole pairs form a bound or anti-bound state, the convergence is achieved quickly. This numeric observation is physically plausible, because for split-off states, the two particles are close to each other and hence the finite size effects become irrelevant. By determining eigen-vectors of the matrix we obtain the $f_{d}$ which can be used to calculate the coherence length via Eq. (19).

Upper and lower edges of the particle-hole continuum (PHC) is obtained by ignoring the 2-particle interactions and diagonalizing $H_{\text {eff }}$ in the basis of $\left|K, K^{\prime}\right\rangle$ which gives rise to free particle-hole energies. Then the maximum (minimum) eigen-value in $K^{\prime}$ for a fixed value of $K$ in Brillouin zone determines the upper (lower) edge of the $\mathrm{PHC}^{28,44}$.

Plain series result of electron-hole excitation spectrum for $t=-0.5, U=0.5$, and $V=0$ is depicted in Fig. 5 This figure shows that there are three singlet and two triplet modes. The singlet channel contains two anti-bound states and one 


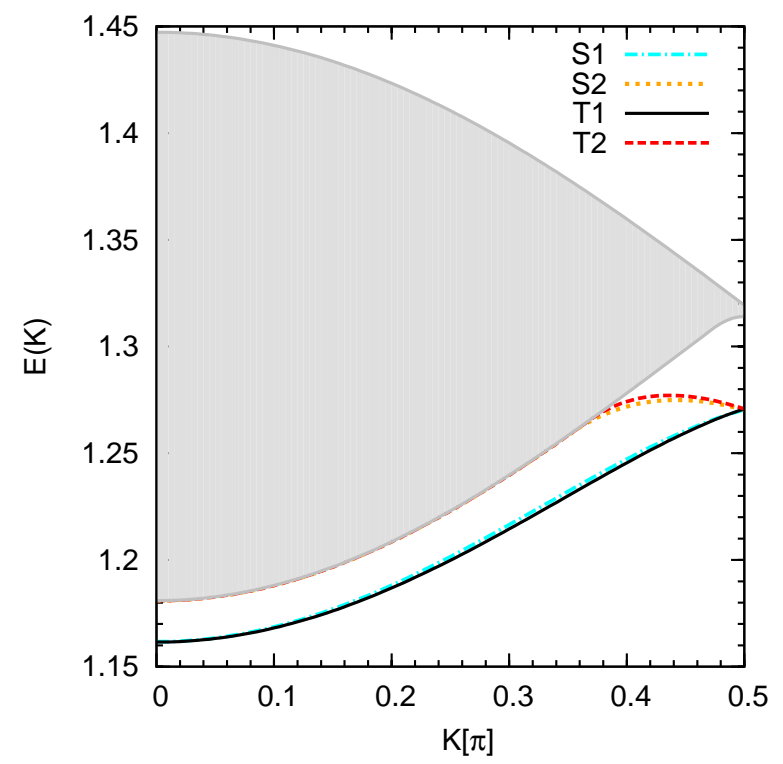

FIG. 7: Plain series result of electron-hole energy versus $K$ for $t=-0.8, U=0.0$, and $V=0.1$. There are two singlet ( $S 1$ and $S 2$ ) and two triplet ( $T 1$ and $T 2$ ) bound state modes. Each singlet is nearly degenerate with one triplet which show the effect of on site interaction $U$ in separation of singlet and triplet state.

bound state mode while the triplet modes are bound states. The two singlet anti-bound state branches and the two triplet branches are degenerate at the edges of the Brillouin zone, $K= \pm \frac{\pi}{2}$. Hence, when longer range Coulomb interaction $(V)$ is absent, on-site interaction $U$ stabilizes the triplet excitations with respect to singlet ones. Note that in the limit $U \rightarrow 0$, a simple band picture would imply the singlet and triplet branches to have the same excitation energies. Therefore such singlet-triplet splitting is a remarkable effect of short range correlations which manifests itself at molecular ${ }^{45}$ as well as infinite size systems ${ }^{46}$ where the correlation effects is believed to play important role.

We have also plotted the inverse of the coherence length in Fig. 6 corresponding to parameter values of Fig. 5. Except for $S 1$ and $S 2$ branches, the coherence length increases with decreasing bounding energy. Coherence lengths of $S 1$ and $S 2$ decrease with decreasing of anti-bounding energy and $S 2$ mode has a discontinuity at $K^{*}=0.46 \pi$. For $S 1$ mode which is anti-bound everywhere in the BZ, the coherence length always remains finite. But for other branches who enter the $\mathrm{PHC}$ at some momentum, the coherence length become infinite at those particular momenta.

Plain series results for electron-hole energy and inverse of coherence length versus $K$ for $t=-0.8, U=0.0$, and $V=0.1$ have been depicted in Figs. 7 and 8 respectively. Fig. 7 shows that there are two singlet and two triplet modes which all of them are bound states. The fact that the all modes are bound state compared with previous case, $V=0$, indicates that the long range part of the Coulomb interaction amounts to attraction among electron-hole pairs in both singlet and triplet channels which in turn forms the so called ex- citonic states. Each of singlet branches is nearly degenerate with the corresponding triplet branch. This again emphasizes that the short range Hubbard interaction $U$ is the major mechanism behind the splitting between singlet and triplet collective mode branches.

In Fig. 8 we display the behavior for coherence length for the same parameter values as in Fig. 7 Coherence length of $S 2$ and $T 2$, that are nearly degenerate, monotonically increases by decreasing $K$ until a certain value $K^{*}$ is reached, beyond which the coherence length becomes infinite. But for $S 1$ and $T 1$ there is always a finite coherence length, indicating the corresponding $\mathrm{p}-\mathrm{h}$ bound state exists everywhere in the BZ.

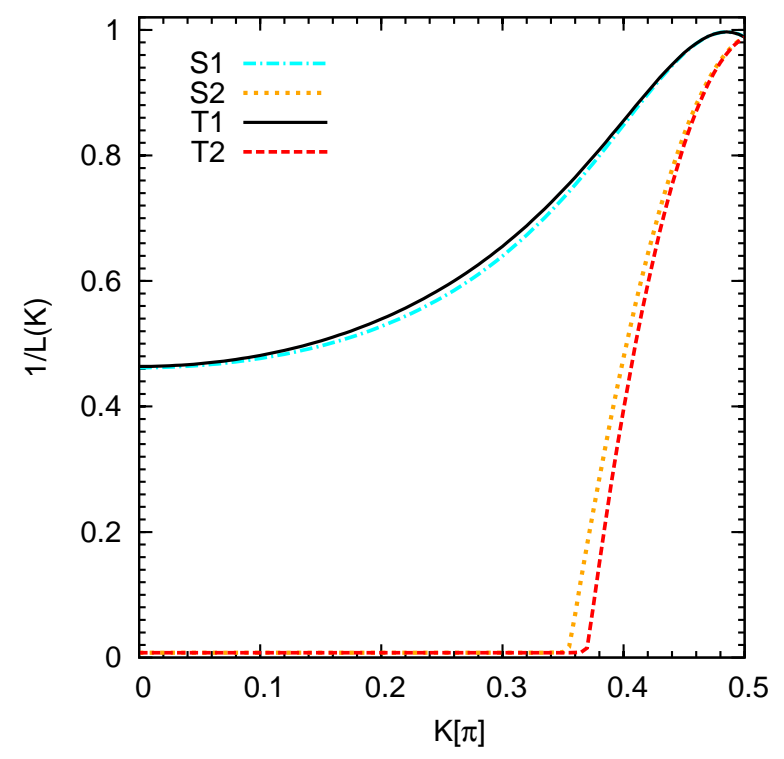

FIG. 8: Inverse of the coherence length versus $K$ for $t=-0.8$, $U=0.0$, and $V=0.1$. Inverse of coherence length is identical for $S 2$ and $T 2$ and increases monotonically with $K$ while for $S 1$ and $T 1$ there is a peak not exact at the border.

Now let us consider a case where both $U$ and $V$ are present. In Figs. 9 and 10, we have plotted the plain series results for particle-hole energy and inverse coherence length versus $K$ for $t=-0.8, U=0.4$, and $V=0.3$. Again the behavior of coherence length and binding energy are in accordance with each other. As can be see while due to presence of non-zero $V$, all modes are binding, still on-site correlation $U$ leads to singlet-triplet splitting as expected. As can be seen in Fig. 9 , near the BZ edge, triplets are lowest lying excitations. But for smaller momentum transfer $K$, there are regions where the singlet mode will become lowest excited state.

In the appendix we give analytic expressions for the electron-hole energy $E_{\mathrm{ph}}(K)$ for singlet and triplet modes at the zone edge, $K=\frac{\pi}{2}$, up to order 8 . These relations are valid for $t^{8} \ll V$. 


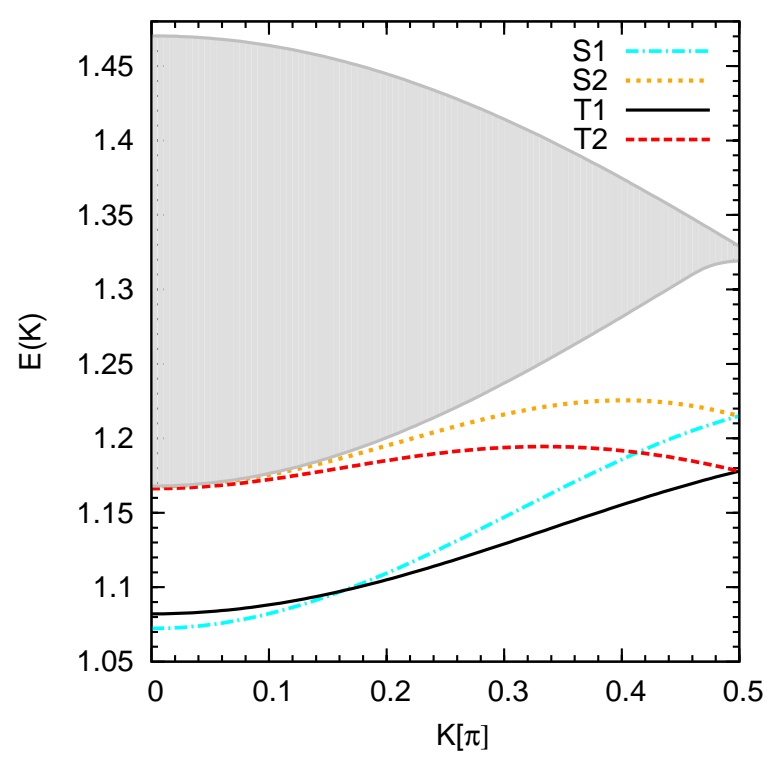

FIG. 9: Plain series result of electron-hole energy versus $K$ for $t=$ $-0.8, U=0.4$, and $V=0.3$. There are two singlet, $S 1$ and $S 2$, and two triplet, $T 1$ and $T 2$, bound state modes. For $K<0.16$ the lowest energy mode is singlet while for $K>0.16$ it is a triplet state. At the border of Brillouin zone, $K= \pm \frac{\pi}{2}$, the two singlets and two triplets are degenerate.

\section{SUMMARY DISCUSSION}

We used perturbative continuous unitary transformation method to study the 1D extended ionic Hubbard model in the band insulating regime. EIHM in addition to the usual ionic Hubbard model includes a n.n. neighbor Coulomb repulsion, $V$. We treated the ionic potential as unperturbed part and studied the effect of hopping and Coulomb interaction terms, perturbatively. The convergence range of the perturbative results were extended by using a Padé scheme to fit the results and extrapolating them to larger values of interaction parameters $U, V$ which enables us to approach the limit where band gap vanishes. Effective Hamiltonian was obtained and diagonalized in zero-, one-particle and particle-hole sectors. Zeroparticle sector gives us the ground state energy which upon using the Hellman-Feynman theorem lead to a perturbative expression for the ionicity. Ionicity in the absence of $U, V$ terms at zero temperature has its maximum value of 1 , which is a characteristic of band insulators. Ionicity decreases by increasing $U$, while by increasing $V$, it tends to increase. Therefore the on-site correlations $U$ are useful to remedy the valence discrepancy in transition metal oxides such as $\mathrm{BaTiO}_{3}$, $\mathrm{KNbO}_{3}$, and $\mathrm{KTaO}_{3}$.

In one-quasi-particle sector we calculated the electron and hole dispersions which was achieved by adding one electron or a hole to the system. Study of the charge gap at $V=0$ indicated that the band gap tends to zero for a critical value of $U$ which is in agreement with state of the art DMRG results ${ }^{14,47}$.

In the particle-hole sector, the total spin of particle-hole pair is a good quantum number which helps to classify particle-

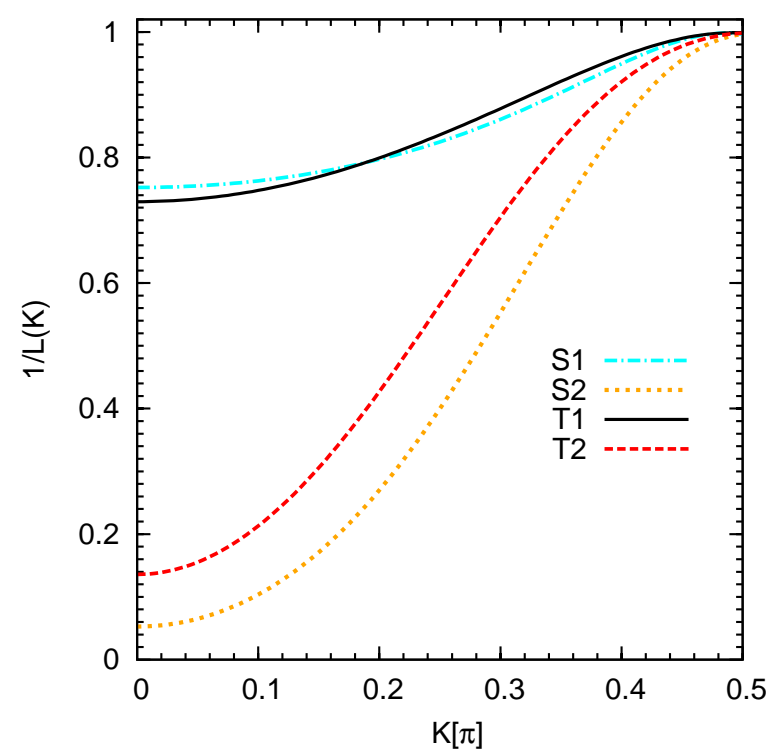

FIG. 10: Inverse of coherence length versus $K$ for $t=-0.8, U=$ 0.4 , and $V=0.3$. There are four graphs correspond to two singlets, $S 1$ and $S 2$, and two triplets, $T 1$ and $T 2$, modes. At the $K= \pm \frac{\pi}{2}$ all states have same coherence length that is equal to 1 .

hole excitations in singlet and triplet channels. The particlehole continuum is characterized by the spectrum of free particle-hole pairs. Turning on the interaction gives rise to split-off (bound or anti-bound) states in the triplet and singlet $\mathrm{PH}$ channels. There are in general three/two singlet and two triplet branches in the reduced Brillouin zone $(-\pi / 2 \leq k<$ $\pi / 2)$. Given that the bound states in each total spin channel are degenerate at $k= \pm \pi / 2$, the two branches can be thought of as a single branch in the larger BZ (corresponding to the $\Delta=0$ limit). The so obtained triplet and singlet correspond to a pole in the appropriate susceptibility when the problem is viewed from itinerant limit. In this sense we can interpret the triplet and singlet split-off states as collective modes. We also discussed the coherence length of these collective states. In general when the binding (anti-binding) energy of the collective states which is defined as the energy difference between the energy eigen-value corresponding to the collective state and the lower (upper) bound of the PHC decreases the coherence length which is a measure of PH bound- (anti-bound-) state increases. At a critical value of center of mass momentum $K$ where the collective mode branch enters the continuum of free particle-hole pairs the coherence length becomes infinitely large. Within PCUT when the $U$ term is zero, for finite $V$ the singlet and triplet collective modes are almost degenerate. The major effect of n.n. Coulomb repulsion $V$ is to render the singlet branches into binding collective states by pushing them below the PHC, while the major role of $U$ is to give rise to remarkable singlet-triplet splitting 46 . Both singlet and triplet states which will be bound states in presence of $V$ can be interpreted as the excitonic states of the band insulator under study. Excitonic states are well-known for their optical signatures. Therefore the PCUT approach seems to be 
promising for investigation of excitonic effects in the optical spectra ${ }^{36.48}$. Within the PCUT formulation it is also possible to discuss the propagation of two-electron and two-hole states which touches upon the problem of superconductivity in strongly correlated electron systems.

\section{ACKNOWLEDGEMENT}

S.A.J. thanks G. S. Uhrig for critical reading of the manuscript and many useful comments and suggestions. This research was supported by the Vice Chancellor for Research Affairs of the Isfahan University of Technology (IUT). S.A.J was supported by the National Elite Foundation (NEF) of Iran.

\section{Appendix}

Ground state energy and hopping terms up to order 9 in the exponent summation of $t, U$, and $V$.

$$
\begin{aligned}
\epsilon_{0}= & -\frac{1}{2}+\frac{1}{2} U-\frac{1}{8} t^{2}+\frac{3}{16} t^{2} V-\frac{1}{8} t^{2} U-\frac{9}{32} t^{2} V^{2}+\frac{3}{8} t^{2} U V-\frac{1}{8} t^{2} U^{2}+\frac{3}{128} t^{4}+\frac{27}{64} t^{2} V^{3}-\frac{27}{32} t^{2} U V^{2} \\
& +\frac{9}{16} t^{2} U^{2} V-\frac{1}{8} t^{2} U^{3}-\frac{1}{8} t^{4} V+\frac{11}{128} t^{4} U-\frac{81}{128} t^{2} V^{4}+\frac{27}{16} t^{2} U V^{3}-\frac{27}{16} t^{2} U^{2} V^{2}+\frac{3}{4} t^{2} U^{3} V-\frac{1}{8} t^{2} U^{4} \\
& +\frac{109}{256} t^{4} V^{2}-\frac{37}{64} t^{4} U V+\frac{49}{256} t^{4} U^{2}-\frac{5}{512} t^{6}+\frac{243}{256} t^{2} V^{5}-\frac{405}{128} t^{2} U V^{4}+\frac{135}{32} t^{2} U^{2} V^{3}-\frac{45}{16} t^{2} U^{3} V^{2}+\frac{15}{16} t^{2} U^{4} V \\
& -\frac{1}{8} t^{2} U^{5}-\frac{2407}{2048} t^{4} V^{3}+\frac{19}{8} t^{4} U V^{2}-\frac{201}{128} t^{4} U^{2} V+\frac{175}{512} t^{4} U^{3}+\frac{97}{1024} t^{6} V-\frac{17}{256} t^{6} U-\frac{729}{512} t^{2} V^{6}+\frac{729}{128} t^{2} U V^{5} \\
& -\frac{1215}{128} t^{2} U^{2} V^{4}+\frac{135}{16} t^{2} U^{3} V^{3}-\frac{135}{32} t^{2} U^{4} V^{2}+\frac{9}{8} t^{2} U^{5} V-\frac{1}{8} t^{2} U^{6}+\frac{23459}{8192} t^{4} V^{4}-\frac{7861}{1024} t^{4} U V^{3}+\frac{7791}{1024} t^{4} U^{2} V^{2} \\
& -\frac{1699}{512} t^{4} U^{3} V+\frac{551}{1024} t^{4} U^{4}-\frac{267}{512} t^{6} V^{2}+\frac{2939}{4096} t^{6} U V-\frac{245}{1024} t^{6} U^{2}+\frac{175}{32768} t^{8}+\frac{2187}{1024} t^{2} V^{7}-\frac{5103}{512} t^{2} U V^{6} \\
& +\frac{5103}{256} t^{2} U^{2} V^{5}-\frac{2835}{128} t^{2} U^{3} V^{4}+\frac{945}{64} t^{2} U^{4} V^{3}-\frac{189}{32} t^{2} U^{5} V^{2}+\frac{21}{16} t^{2} U^{6} V-\frac{1}{8} t^{2} U^{7}-\frac{210403}{32768} t^{4} V^{5} \\
& +\frac{175677}{8192} t^{4} U V^{4}-\frac{58025}{2048} t^{4} U^{2} V^{3}+\frac{38025}{2048} t^{4} U^{3} V^{2}-\frac{3093}{512} t^{4} U^{4} V+\frac{1599}{2048} t^{4} U^{5}+\frac{70447}{32768} t^{6} V^{3}-\frac{35913}{8192} t^{6} U V^{2} \\
& +\frac{23841}{8192} t^{6} U^{2} V-\frac{81}{128} t^{6} U^{3}-\frac{1257}{16384} t^{8} V+\frac{1787}{32768} t^{8} U
\end{aligned}
$$

$$
\begin{aligned}
t_{0, e}= & t_{0, h}+U+2 V=\frac{1}{2}+2 V+\frac{1}{8} t^{2}-\frac{3}{8} t^{2} V+\frac{1}{4} t^{2} U+\frac{3}{4} t^{2} V^{2}-t^{2} U V+\frac{1}{4} t^{2} U^{2}-\frac{3}{128} t^{4}-\frac{21}{16} t^{2} V^{3} \\
& +\frac{21}{8} t^{2} U V^{2}-\frac{3}{2} t^{2} U^{2} V+\frac{1}{4} t^{2} U^{3}+\frac{1}{4} t^{4} V-\frac{11}{64} t^{4} U+\frac{69}{32} t^{2} V^{4}-\frac{23}{4} t^{2} U V^{3}+\frac{21}{4} t^{2} U^{2} V^{2}-2 t^{2} U^{3} V \\
& +\frac{1}{4} t^{2} U^{4}-\frac{37}{32} t^{4} V^{2}+\frac{99}{64} t^{4} U V-\frac{53}{128} t^{4} U^{2}+\frac{5}{512} t^{6}-\frac{219}{64} t^{2} V^{5}+\frac{365}{32} t^{2} U V^{4}-\frac{115}{8} t^{2} U^{2} V^{3}+\frac{35}{4} t^{2} U^{3} V^{2} \\
& -\frac{5}{2} t^{2} U^{4} V+\frac{1}{4} t^{2} U^{5}+\frac{7769}{2048} t^{4} V^{3}-\frac{1927}{256} t^{4} U V^{2}+\frac{563}{128} t^{4} U^{2} V-\frac{393}{512} t^{4} U^{3}-\frac{97}{512} t^{6} V+\frac{17}{128} t^{6} U+\frac{681}{128} t^{2} V^{6} \\
& -\frac{681}{32} t^{2} U V^{5}+\frac{1095}{32} t^{2} U^{2} V^{4}-\frac{115}{4} t^{2} U^{3} V^{3}+\frac{105}{8} t^{2} U^{4} V^{2}-3 t^{2} U^{5} V+\frac{1}{4} t^{2} U^{6}-\frac{84403}{8192} t^{4} V^{4}+\frac{55553}{2048} t^{4} U V^{3} \\
& -\frac{50827}{2048} t^{4} U^{2} V^{2}+\frac{19}{2} t^{4} U^{3} V-\frac{1265}{1024} t^{4} U^{4}+\frac{5843}{4096} t^{6} V^{2}-\frac{3941}{2048} t^{6} U V+\frac{275}{512} t^{6} U^{2}-\frac{175}{32768} t^{8}-\frac{2091}{256} t^{2} V^{7} \\
& +\frac{4879}{128} t^{2} U V^{6}-\frac{4767}{64} t^{2} U^{2} V^{5}+\frac{2555}{32} t^{2} U^{3} V^{4}-\frac{805}{16} t^{2} U^{4} V^{3}+\frac{147}{8} t^{2} U^{5} V^{2}-\frac{7}{2} t^{2} U^{6} V+\frac{1}{4} t^{2} U^{7} \\
& +\frac{814169}{32768} t^{4} V^{5}-\frac{1337047}{16384} t^{4} U V^{4}+\frac{208933}{2048} t^{4} U^{2} V^{3}-\frac{251523}{4096} t^{4} U^{3} V^{2}+\frac{35873}{2048} t^{4} U^{4} V-\frac{3725}{2048} t^{4} U^{5} \\
& -\frac{231121}{32768} t^{6} V^{3}+\frac{115041}{8192} t^{6} U V^{2}-\frac{68499}{8192} t^{6} U^{2} V+\frac{769}{512} t^{6} U^{3}+\frac{1257}{8192} t^{8} V-\frac{1787}{16384} t^{8} U
\end{aligned}
$$




$$
\begin{aligned}
t_{2}= & \left(\frac{1}{16}-\frac{1}{16} V+\frac{1}{16} U+\frac{1}{16} V^{2}-\frac{1}{8} U V+\frac{1}{16} U^{2}-\frac{1}{64} t^{2}-\frac{1}{16} V^{3}+\frac{3}{16} U V^{2}-\frac{3}{16} U^{2} V+\frac{1}{16} U^{3}\right. \\
& +\frac{9}{128} t^{2} V-\frac{1}{16} t^{2} U+\frac{1}{16} V^{4}-\frac{1}{4} U V^{3}+\frac{3}{8} U^{2} V^{2}-\frac{1}{4} U^{3} V+\frac{1}{16} U^{4}-\frac{99}{512} t^{2} V^{2}+\frac{165}{512} t^{2} U V-\frac{9}{64} t^{2} U^{2} \\
& +\frac{15}{2048} t^{4}-\frac{1}{16} V^{5}+\frac{5}{16} U V^{4}-\frac{5}{8} U^{2} V^{3}+\frac{5}{8} U^{3} V^{2}-\frac{5}{16} U^{4} V+\frac{1}{16} U^{5}+\frac{879}{2048} t^{2} V^{3}-\frac{2113}{2048} t^{2} U V^{2} \\
& +\frac{889}{1024} t^{2} U^{2} V-\frac{1}{4} t^{2} U^{3}-\frac{135}{2048} t^{4} V+\frac{117}{2048} t^{4} U+\frac{1}{16} V^{6}-\frac{3}{8} U V^{5}+\frac{15}{16} U^{2} V^{4}-\frac{5}{4} U^{3} V^{3}+\frac{15}{16} U^{4} V^{2} \\
& -\frac{3}{8} U^{5} V+\frac{1}{16} U^{6}-\frac{6965}{8192} t^{2} V^{4}+\frac{21737}{8192} t^{2} U V^{3}-\frac{13}{4} t^{2} U^{2} V^{2}+\frac{3721}{2048} t^{2} U^{3} V-\frac{25}{64} t^{2} U^{4}+\frac{20605}{65536} t^{4} V^{2} \\
& -\frac{1037}{2048} t^{4} U V+\frac{3449}{16384} t^{4} U^{2}-\frac{35}{8192} t^{6}-\frac{1}{16} V^{7}+\frac{7}{16} U V^{6}-\frac{21}{16} U^{2} V^{5}+\frac{35}{16} U^{3} V^{4}-\frac{35}{16} U^{4} V^{3}+\frac{21}{16} U^{5} V^{2} \\
& -\frac{7}{16} U^{6} V+\frac{1}{16} U^{7}+\frac{51655}{32768} t^{2} V^{5}-\frac{197589}{32768} t^{2} U V^{4}+\frac{157273}{16384} t^{2} U^{2} V^{3}-\frac{64179}{8192} t^{2} U^{3} V^{2}+\frac{13425}{4096} t^{2} U^{4} V \\
& \left.-\frac{9}{16} t^{2} U^{5}-\frac{72489}{65536} t^{4} V^{3}+\frac{41967}{16384} t^{4} U V^{2}-\frac{33523}{16384} t^{4} U^{2} V+\frac{4557}{8192} t^{4} U^{3}+\frac{493}{8192} t^{6} V-\frac{53}{1024} t^{6} U\right) t^{2}
\end{aligned}
$$

$$
\begin{aligned}
t_{4}= & \left(-\frac{1}{256}+\frac{1}{64} V-\frac{3}{256} U-\frac{85}{2048} V^{2}+\frac{1}{16} U V-\frac{3}{128} U^{2}+\frac{3}{1024} t^{2}+\frac{47}{512} V^{3}-\frac{425}{2048} U V^{2}+\frac{5}{32} U^{2} V\right. \\
& -\frac{5}{128} U^{3}-\frac{3}{128} t^{2} V+\frac{9}{512} t^{2} U-\frac{1499}{8192} V^{4}+\frac{141}{256} U V^{3}-\frac{1275}{2048} U^{2} V^{2}+\frac{5}{16} U^{3} V-\frac{15}{256} U^{4}+\frac{881}{8192} t^{2} V^{2} \\
& -\frac{329}{2048} t^{2} U V+\frac{15}{256} t^{2} U^{2}-\frac{35}{16384} t^{4}+\frac{2797}{8192} V^{5}-\frac{10493}{8192} U V^{4}+\frac{987}{512} U^{2} V^{3}-\frac{2975}{2048} U^{3} V^{2}+\frac{35}{64} U^{4} V \\
& \left.-\frac{21}{256} U^{5}-\frac{24475}{65536} t^{2} V^{3}+\frac{13637}{16384} t^{2} U V^{2}-\frac{1247}{2048} t^{2} U^{2} V+\frac{75}{512} t^{2} U^{3}+\frac{55}{2048} t^{4} V-\frac{83}{4096} t^{4} U\right) t^{4}
\end{aligned}
$$

$$
\begin{aligned}
t_{6}= & \left(\frac{1}{2048}-\frac{7}{2048} V+\frac{5}{2048} U+\frac{951}{65536} V^{2}-\frac{21}{1024} U V+\frac{15}{2048} U^{2}-\frac{5}{8192} t^{2}-\frac{6345}{131072} V^{3}+\frac{6657}{65536} U V^{2}\right. \\
& \left.-\frac{147}{2048} U^{2} V+\frac{35}{2048} U^{3}+\frac{111}{16384} t^{2} V-\frac{5}{1024} t^{2} U\right) t^{6}
\end{aligned}
$$

$$
t_{8}=\left(-\frac{5}{65536}+\frac{25}{32768} V-\frac{35}{65536} U\right) t^{8}
$$

Electron-hole energy $E(K)$ up to order 8 at $K=\frac{\pi}{2}$ valid for $t^{8} \ll V$.

$$
\begin{aligned}
E_{S 1}\left(\frac{\pi}{2}\right)= & E_{S 2}\left(\frac{\pi}{2}\right)=1+\frac{3}{2} V-U+\frac{1}{4} t^{2}-\frac{5}{8} t^{2} V+\frac{5}{8} t^{2} U+\frac{17}{16} t^{2} V^{2}-\frac{13}{8} t^{2} U V+\frac{3}{8} t^{2} U^{2}-\frac{3}{64} t^{4}-\frac{53}{32} t^{2} V^{3} \\
& +\frac{111}{32} t^{2} U V^{2}-\frac{33}{16} t^{2} U^{2} V+\frac{5}{8} t^{2} U^{3}+\frac{217}{512} t^{4} V-\frac{27}{64} t^{4} U+\frac{161}{64} t^{2} V^{4}-\frac{109}{16} t^{2} U V^{3}+\frac{105}{16} t^{2} U^{2} V^{2} \\
& -\frac{13}{4} t^{2} U^{3} V+\frac{3}{8} t^{2} U^{4}-\frac{3357}{2048} t^{4} V^{2}+\frac{319}{128} t^{4} U V-\frac{81}{128} t^{4} U^{2}+\frac{5}{256} t^{6}-\frac{485}{128} t^{2} V^{5}+\frac{1625}{128} t^{2} U V^{4} \\
& -\frac{535}{32} t^{2} U^{2} V^{3}+\frac{185}{16} t^{2} U^{3} V^{2}-\frac{55}{16} t^{2} U^{4} V+\frac{5}{8} t^{2} U^{5}+\frac{38801}{8192} t^{4} V^{3}-\frac{10115}{1024} t^{4} U V^{2}+\frac{3067}{512} t^{4} U^{2} V \\
& -\frac{201}{128} t^{4} U^{3}-\frac{667}{2048} t^{6} V+\frac{655}{2048} t^{6} U-\frac{1}{4096} \frac{t^{8}}{V}+\frac{1457}{256} t^{2} V^{6}-\frac{2919}{128} t^{2} U V^{5}+\frac{4845}{128} t^{2} U^{2} V^{4}-\frac{545}{16} t^{2} U^{3} V^{3} \\
& +\frac{525}{32} t^{2} U^{4} V^{2}-\frac{39}{8} t^{2} U^{5} V+\frac{3}{8} t^{2} U^{6}-\frac{386537}{32768} t^{4} V^{4}+\frac{65089}{2048} t^{4} U V^{3}-\frac{31179}{1024} t^{4} U^{2} V^{2}+\frac{7177}{512} t^{4} U^{3} V \\
& -\frac{477}{256} t^{4} U^{4}+\frac{16737}{8192} t^{6} V^{2}-\frac{12677}{4096} t^{6} U V+\frac{1701}{2048} t^{6} U^{2}-\frac{147}{16384} t^{8}-\frac{7}{4096} \frac{t^{8} U}{V}
\end{aligned}
$$




$$
\begin{aligned}
E_{T 1}\left(\frac{\pi}{2}\right)= & E_{T 2}\left(\frac{\pi}{2}\right)=1+\frac{3}{2} V-U+\frac{1}{4} t^{2}-\frac{5}{8} t^{2} V+\frac{3}{8} t^{2} U+\frac{9}{8} t^{2} V^{2}-\frac{11}{8} t^{2} U V+\frac{3}{8} t^{2} U^{2}-\frac{3}{64} t^{4}-\frac{59}{32} t^{2} V^{3} \\
& +\frac{111}{32} t^{2} U V^{2}-\frac{33}{16} t^{2} U^{2} V+\frac{3}{8} t^{2} U^{3}+\frac{217}{512} t^{4} V-\frac{17}{64} t^{4} U+\frac{93}{32} t^{2} V^{4}-\frac{119}{16} t^{2} U V^{3}+\frac{111}{16} t^{2} U^{2} V^{2} \\
& -\frac{11}{4} t^{2} U^{3} V+\frac{3}{8} t^{2} U^{4}-\frac{3597}{2048} t^{4} V^{2}+\frac{283}{128} t^{4} U V-\frac{81}{128} t^{4} U^{2}+\frac{5}{256} t^{6}-\frac{575}{128} t^{2} V^{5}+\frac{1865}{128} t^{2} U V^{4} \\
& -\frac{595}{32} t^{2} U^{2} V^{3}+\frac{185}{16} t^{2} U^{3} V^{2}-\frac{55}{16} t^{2} U^{4} V+\frac{3}{8} t^{2} U^{5}+\frac{43969}{8192} t^{4} V^{3}-\frac{10487}{1024} t^{4} U V^{2}+\frac{3163}{512} t^{4} U^{2} V \\
& -\frac{149}{128} t^{4} U^{3}-\frac{667}{2048} t^{6} V+\frac{433}{2048} t^{6} U-\frac{1}{4096} \frac{t^{8}}{V}+\frac{879}{128} t^{2} V^{6}-\frac{3453}{128} t^{2} U V^{5}+\frac{5595}{128} t^{2} U^{2} V^{4}-\frac{595}{16} t^{2} U^{3} V^{3} \\
& +\frac{555}{32} t^{2} U^{4} V^{2}-\frac{33}{8} t^{2} U^{5} V+\frac{3}{8} t^{2} U^{6}-\frac{456009}{32768} t^{4} V^{4}+\frac{73161}{2048} t^{4} U V^{3}-\frac{34055}{1024} t^{4} U^{2} V^{2}+\frac{6777}{512} t^{4} U^{3} V \\
& -\frac{477}{256} t^{4} U^{4}+\frac{18031}{8192} t^{6} V^{2}-\frac{11619}{4096} t^{6} U V+\frac{1725}{2048} t^{6} U^{2}-\frac{147}{16384} t^{8}-\frac{5}{4096} \frac{t^{8} U}{V}
\end{aligned}
$$

1 J. Hubbard, and J. B. Torrance, Phys. Rev. Lett. 47, 1750 (1981).

2 J. B. Torrance, J. E. Vazquez, J. J. Mayerle, and V. Y. Lee, Phys. Rev. Lett. 46, 253 (1980).

3 J. B. Torrance, A. Girlando, J. J. Mayerle, J. I. Crowley, V. Y. Lee, and P. Batail, Phys. Rev. Lett. 47, 1747 (1981).

4 Y. Tokura, T. Koda, T. Mitani, and G. Saito, Solid State Commun. 43, 757 (1982).

5 T. Mitani, G. Saito, Y. Tokura, and T. Koda, Phys. Rev. Lett. 20, 842 (1984).

${ }^{6}$ N. Nagaosa and J. Takimoto, J. Phys. Soc. Jpn. 55, 2735 (1986).

7 N. Nagaosa and J. Takimoto, J. Phys. Soc. Jpn. 55, 2745 (1986).

8 A. Avignon C. A. Balseiro C. R. Proetto, and B. Alascio, Phys. Rev. B 33, 205 (1986).

9 R. E. Cohen and H. Krakauer, Phys. Rev. B 42, 6416 (1990).

10 T. Neumman, G. Borstel, C. Scharfschwerdt, and M. Neumman, Phys. Rev. B 46, 10623 (1992).

11 T. Egami, S. Ishihara, and M. Tachiki, Science 261, 1307 (1993).

12 M. Fabrizio,A. O. Gogolin, and A. A. Nersesyan, Phys. Rev. Lett. 83, 2014 (1999).

13 C. D. Batista and A. A. Aligia, Phys. Rev. Lett. 92, 246405 (2004).

14 S. R. Manmana, V. Meden, R. M. Noack, and K. Schonhammer, Phys. Rev. B 70, 155115 (2004).

15 A. Garg, H. R. Krishnamurthy, and M. Randeria, Phys. Rev. Lett. 97, 046403 (2006).

${ }^{16}$ K. Byczuk, M. Sekania, W. Hofstetter, and A. P. Kampf, Phys. Rev. B 79, 121103R (2009).

17 M. Hafez, S. A. Jafari, and M. R. Abolhassani, Phys. Lett. A 373, 4479 (2009).

18 N. Paris, K. Bouadim, F. Hebert, G. G. Batrouni, and R. T. Scalettar, Phys. Rev. Lett. 98, 046403 (2007).

${ }^{19}$ K. Bouadim, N. Paris, F. Hebert, G. G. Batrouni, and R. T. Scalettar, Phys. Rev. B 76, 085112 (2007).

${ }^{20}$ M. Hafez, S. A. Jafari, Sh. Adibi, and F. Shahbazi, Phys. Rev. B 81245131 (2010).

${ }^{21}$ N. Maeshima and K. Yonemitsu, J. Phys.: Conf. Series 21, 183 (2005).

${ }^{22}$ M. Ono, K. Miura, A. Maeda, H. Matsuzaki, H. Kishida, Y. Taguchi, Y. Tokura, M. Yamashita, and H. Okamoto, Phys. Rev. B 70, 085101 (2004).
${ }^{23}$ I. Batistic and A. R. Bishop, Phys. Rev. B 45, 5282 (1992).

${ }^{24}$ S. Iwai, M. Ono, A. Maeda, H. Matsuzaki, H. Kishida, H. Okamoto, and Y. Tokura, Phys. Rev. Lett. 91, 057401 (2003).

25 J. Ohara and Sh. Yamamoto, Phys. Rev. B 73, 045122 (2006).

${ }^{26}$ C. Knetter, and G. S. Uhrig, Eur. Phys. J. B 13, 209 (2000).

${ }^{27}$ C. Knetter, K. P. Schmidt, and G. S. Uhrig, J. Phys. A 36, 7889 (2003).

28 C. Knetter, K. P. Schmidt, and G. S. Uhrig, Eur. Phys. J. B 36, 525 (2003).

29 T. Fischer, S. Duffe, G. S. Uhrig, New J. Phys. 12, 033048 (2010).

30 F. Wegner Ann. Phys. (Leipzing) 3, 77 (1994).

31 A. Mielke, Eur. Phys. J. B 5, 605 (1998).

${ }^{32}$ M. P. Gelfand, and R. R. P. Singh, Adv. Phys. 49, 93 (2000).

33 http://t1.physik.tu-dortmund.de/uhrig

34 M. Arlego, and W. Brenig. Eur. Phys. J. B 53, 193 (2006).

35 W. Brenig, and M. Grzeschik, Phys. Rev. B 69, 064420 (2004).

${ }^{36}$ K. P. Schmidt, C. Knetter, and G. S. Uhrig, Phys. Rev. B 69, 104417 (2004).

37 C. Knetter, and G. S. Uhrig, Phys. Rev. Lett. 92, 027204 (2004).

38 K. P. Schmidt, and G. S. Uhrig, Phys. Rev. B 75, 224414 (2007).

39 J. Vidal, S. Dusuel, and K. P. Schmidt, Phys. Rev. B 79, 033109 (2009).

40 S. Dusuel, M. Kamfor, K. P. Schmidt, R. Thomale, and J. Vidal, Phys. Rev. B 81, 064412 (2010).

${ }^{41}$ H. Y. Yang, A. Laeuchli, F. Mila, and K. P. Schmidt, arXiv:1006.5649

42 K. P. Schmidt et al, Phys. Rev. B 72, 094419 (2005).

43 C. P. Heidbrink, and G. S. Uhrig, Eru. Phys. J. B 30, 443 (2002).

${ }^{44}$ W. Zheng, C. J. Hamer, R. R. P. Singh, S. Trebst, and H. monien, Phys. Rev. B 63, 144410 (2001).

45 E. C. da Silva, J. Gerratt, D. L. Cooper, M. Raimondi, J. Chem. Phys. 101 (1994) 3866, and references therein.

46 G. Baskaran, S.A. Jafari, Phys. Rev. Lett. 89, 016402 (2002); M. Ebrahimkhas, S. A. Jafari, Phys. Rev. B, 79, 205425 (2009).

47 Fei Ye, Bing-Shen Wang, Jizhong Lou, and Zha-Bin Su, Phys. Rev. B 72233409 (2005).

48 K.P. Schmidt and G.S. Uhrig, Mod. Phys. Lett. B 191179 (2005). 